\title{
Primary Breast Osteosarcoma; a case report and review of the literature
}

\author{
Ramesh Omranipour ${ }^{1}$, Fereshteh Ensani ${ }^{1}$, and Maryam Hassanesfahani ${ }^{1}$ \\ ${ }^{1}$ Tehran University of Medical Sciences
}

June 4, 2021

\begin{abstract}
Primary breast osteosarcoma (PBOS) is an extremely rare and poor prognostic malignancy that has not a definitive treatment guideline. Here we presented a successfully treated case of PBOS and provided a comprehensive review of the literature which revealed the divergence of opinions regarding the histogenesis and management of this malignancy.
\end{abstract}

\section{Introduction}

Primary breast sarcomas are very rare, and primary breast osteosarcoma (PBOS) is still far less common. To the best of our knowledge, less than 150 cases of PBOS have been reported by this time; however, based on recent investigations, it is possible that even many of those cases were not really PBOS and they were some variants of metaplastic breast carcinoma. Indeed, they were considered as PBOS because of the lack of conducting a comprehensive histological and immunohistochemical (IHC) evaluation ${ }^{(1)}$. Since there is no common consensus regarding the management of this specific kind of malignancy, reporting each case and its challenges could be helpful to provide more information about this type of aggressive and poor prognostic tumor.

\section{Case Presentation}

A 48-year-old otherwise healthy Caucasian woman presented with two weeks history of a painless lump in the right breast. There was neither history of trauma nor chest wall irradiation, nor a previous history of a benign or malignant lesion in the breast. No screening mammography had been performed by that time. Physical examination revealed an ill-defined, firm, mobile, and nontender six $\mathrm{cm}$ mass in the central and lateral portion of the breast, behind the nipple-areola complex.

The physical exam of the axillary area and contralateral breast were unremarkable.

The mammography and ultrasonography revealed an irregular bulky mass with a lobulated border in the lateral part of the right breast. On MRI study, there was a $4 \mathrm{~cm}$ mass in the lateral part of the right breast with a high signal intensity at the periphery of the tumor.

The initial Core needle biopsy findings were compatible with extra-skeletal osteosarcoma which was confirmed by the second opinion based on the exclusion of the phyllodes tumor and metaplastic carcinoma along with performing IHC that was strongly positive for vimentin with $30-35 \%$ proliferative activity (Ki 67 ) and nonreactive for Pan-Ck.

There was no evidence of distant metastasis based on liver function test, chest and abdominal CT, and bone scan.

As our institute routine, the patient was discussed in a multidisciplinary team and a simple mastectomy followed by Adriamycin and Ifosfamide regimen of chemotherapy and 50 Gy radiation was planned for her. 
The patient underwent a simple mastectomy. Although the tumor was not grossly fixed to the underlying chest wall structures, the deep margin was too close to the fascia of the pectoralis muscle. Therefore, a thin discoid shape layer of pectoral muscle just beneath the tumor lodge was resected en-bloc with the rest of the specimen. Additionally, four enlarged lymph nodes were resected as a caution. All parts of the specimen were sectioned into $4 \mu \mathrm{m}$ thick and were stained with hematoxylin and eosin as well as Elastica van Gieson. Histological evaluation of the surgical specimen showed atypical tumor cells that were embedded in extensive ossified vermiform plexus of osteoid, bearing necrosis, and autolysis. There was no chondroid differentiation nor evidence of phyllodes tumor or metaplastic carcinoma. The histological result was confirmed by IHC with the avidin-biotin-peroxidase complex method leading to negativity for Pan-CK and CAM 5.2 as well as strong positivity for Vimentin in addition to 30-35\% proliferative activity (Ki67). All margins and lymph nodes were free of tumor. It is worth mentioning that all the histological evaluations have been done by two expert pathologists in the field of sarcoma separately.

After recovery, the patient underwent chemotherapy with the mentioned regimen for five cycles and subsequently received radiotherapy 50 Gy. The treatment plan finished uneventfully last month.

\section{Discussion and Review of the Literature}

\section{Epidemiology and incidence}

Primary breast sarcomas accounting $0.0006 \%$ - $1 \%$ of all breast malignancies and PBOS is far less common which accounts for about $4-12.5 \%$ of primary breast sarcomas ${ }^{(2,3)}$.

There is a discrepancy among the reports in the literature as three large studies of major referral centers over the same span of 40 to 50 years reported different incidences ${ }^{(2,3,4)}$. Two studies from Md Anderson (4) and Mayo clinic ${ }^{(3)}$ reported one to two cases of primary breast osteosarcoma among all sarcomas that they encountered in the same time window (almost 40-50 years); Meanwhile, the third study from Armed Forces Institute of Pathology in Washington DC has reported a much higher incidence of 50 cases within the almost the same period of 40 years ${ }^{(2)}$.

Although PBOS has been reported in a wide range of ages from 16 years old teens ${ }^{(5)}$ to 96 years old patients ${ }^{(6)}$, it usually affects menopaused women in their sixties to eighties ${ }^{(2,3,4)}$.

\section{predisposing and precipitating factors}

the previous history of burn has been noted in one case of PBOS (6) but to the best of our knowledge, it has not been reported elsewhere. Prior history of epithelial breast cancer in the same side or contralateral side has been reported several times ${ }^{(7,8)}$. Some reported cases develop PBOS after irradiation ${ }^{(9,10)}$. In addition, some cases presented with a history of trauma or even a foreign body ${ }^{(2)}$.

\section{Presentation}

On almost all occasions the patients present with a palpable lump; in addition, they usually present as a slowly growing painless lump ${ }^{(2,3,4)}$ even about six years ${ }^{(11)}$, it has reported as a rapidly enlarging mass ${ }^{(12)}$. Regarding the size, it can be as large as $30 \mathrm{~cm}^{(2)}$ or $12 \mathrm{~cm}^{(3)}$ but the average of their size is around $5.5^{(3)}$.

Sites of origin: before labeling them as a primary mammary gland tumor, other neighbor's origins should be ruled out such as underlying ribs, sternum, and even the pectoralis muscle which has been reported by Orta et al ${ }^{(13)}$. It is worth mentioning that there is always a possibility for breast osteosarcoma to be a metastasis from primary bone osteosarcoma ${ }^{(14,15)}$.

\section{Histogenesis}

We believe that not only the rarity of PBOS but also not having enough evidence and consensus about its histogenesis have led to the lack of agreement among the management. Therefore, here we will review some of the literature in detail in order to talk about the histogenesis and the origin of the PBOS. In 2000, Hellmen et al conducted an animal study on dogs and showed that mammary spindle cell tumors and osteosarcomas are derived from pluripotent stem cells ${ }^{(16)}$. In this regard, some authors believe PBOS is originated from 
totipotential stem cells in the mammary gland $(2,16)$ as it can happen after radiation to the chest wall without any significant previous lesion of trauma ${ }^{(9,10)}$. In a different circumstance, there are other studies that mentioned the origin of these specific types of breast sarcoma can be the result of metaplasia, either metaplastic transformation in a pre-existing malignant lesion or non-malignant ones ${ }^{(17,18,19,20)}$. Meanwhile, there are some papers that emphasized the type of pre-existing lesion such as an epithelial cancerous lesion ${ }^{(1,21)}$, an intraductal papilloma ${ }^{(22)}$, previous existing of a fibroadenoma ${ }^{(2,23)}$, or more frequently, phyllodes tumor has been introduced as the pre-existing lesion ${ }^{(24,25,26,27,28,29,30)}$. Diversely, there is a comprehensive investigation that has been published in 2012 by Emad A. Rakha that showed almost all PBOS are derived from the epithelial origin after being under the metaplastic transformation ${ }^{(1)}$.

\section{Diagnostic workup}

There is no doubt regarding the importance of mammography and sonography as the first steps of evaluation of a breast lump. However, as usual, these tumors would present similar to the benign lesions on these modalities ${ }^{(2,31,32)}$, some other evaluation may be needed. In the case of evidence for PBOS on core needle biopsy, in addition to the routine workup of breast cancer, some other assessments such as bone scan and serum alkaline phosphatase activity are recommended by some authors ${ }^{(33,34,35)}$. Furthermore, there is a suggestion to use serum alkaline phosphatase activity for follow-up and monitoring purposes ${ }^{(35)}$. MRI has been used for additional evaluation and information; meanwhile, Dynamic Contrast-Enhanced Magnetic Resonance Imaging, Diffusion-Weighted Imaging Findings, and Proton Spectroscopy have revealed novel findings ${ }^{(36)}$.

\section{Treatment}

There is no general and comprehensive consensus on the management of PBOS. Some consider it as a sarcoma and emphasized that the management of PBOS should be similar to that of other sarcomas ${ }^{(20,37)}$; whereas, some others believe that it should be treated like triple-negative epithelial carcinomas ${ }^{(1)}$. The value and effectiveness of chemotherapy have been emphasized, particularly with the tumor size more than $5 \mathrm{~cm}^{(38,39,40)}$; however, there are still some reports that they did not offer chemotherapy to/for their patients ${ }^{(22,31)}$ even with the tumor size of $6 \mathrm{~cm}^{(41)}$. Although Axillary Lymph Node Dissection (ALND) or sentinel lymph node biopsy has been performed in some reports $(18,22,23,40,42,43)$ in most of the reports ALND was not performed for the patients $(2,3,4,24,44,45)$. Similar to chemotherapy, considering irradiation as a part of treatment is not widely accepted at least as far as the tumor size is not large enough and the margins are clear

from tumoral deposits ${ }^{(4,31,38)}$; however, chest wall irradiation has been suggested by some other authors to reduce the risk of local recurrence as a routine part of treatment ${ }^{(46)}$. It seems that achieving a negative margin either with wide local excision or simple mastectomy without ALND is widely accepted/adapted and offering chemotherapy and radiotherapy should be based on prognostic factors of each patient. It is worth mentioning that due to the local recurrence and distant metastasis rate of about $40 \%$ within the first year ${ }^{(2,47)}$, an aggressive approach including appropriate surgery and adjuvant therapy should be considered while administration of chemotherapy or radiotherapy must be balanced against the consequences of these treatments per case.

\section{Prognostic and predictive factors}

The estimation of 5 and 10- year survival is $38 \%$ and $32 \%$ respectively ${ }^{(2)}$. For overall survival, among many factors such as patient's age at the presentation, tumor size, histopathologic grade and subtype, atypia, mitotic activity, type and extent of surgery, and surgical margin status, only the tumor size less than $5 \mathrm{~cm}$ and fibroblastic subtype have been more accepted as favorable factors and most reliable ones among many of the $\operatorname{series}^{(1,2,3,48)}$. Furthermore, surgical margin status and the type of surgery (local excision vs mastectomy) can be predictive factors for local recurrence. The most common sites for metastasis are the lung, bone, skin, and heart consequently ${ }^{(2,20,26,41,49)}$.

\section{Conclusion}

PBOS is an extremely rare malignancy with divergence among authors' opinions regarding its histogenesis 
and management. Uncertainty about the origin of PBOS and whether it is a metaplastic transformation of epithelial cells or pre-existing lesions such as phyllodes tumors or even arising directly from totipotential stem cells are part of the scenario that has made the dilemma even worse.

In addition, there is not a comprehensive and widely accepted management guideline for its management. Some of the cases received all three parts of possible treatments including surgery, chemotherapy, and radiotherapy; however, there are so many cases that received only one item.

We believe that the most important reason for this discrepancy is not having an agreement about the origin of the tumor; as a result, many authors have approached this tumor similar to a sarcoma while others have tried to manage it like an epithelial-originated malignancy.

Using a comprehensive IHC panel and meticulous pathological evaluation of the tumor would be helpful to find the best plan of treatment meanwhile it is worth mentioning that reporting each case and publishing them would be valuable to collect more information about this specific type of malignancy.

\section{Data availability statement}

The data that support the findings of this study are available on request from the corresponding author. The data are not publicly available due to privacy or ethical restrictions.

\section{References}

1. Rakha EA, Tan PH, Shaaban A, et al. Do primary mammary osteosarcoma and chondrosarcoma exist? A review of a large multi-institutional series of malignant matrix-producing breast tumours. Breast . 2013;22(1):13-18. doi:10.1016/j.breast.2012.09.010

2. Silver SA, Tavassoli FA. Primary osteogenic sarcoma of the breast: a clinicopathologic analysis of 50 cases. Am J Surg Pathol . 1998;22(8):925-933. doi:10.1097/00000478-199808000-00002

3. Adem C, Reynolds C, Ingle JN, Nascimento AG. Primary breast sarcoma: clinicopathologic series from the Mayo Clinic and review of the literature. Br J Cancer . 2004;91(2):237-241. doi:10.1038/sj.bjc.6601920

4. Barrow BJ, Janjan NA, Gutman H, et al. Role of radiotherapy in sarcoma of the breast-a retrospective review of the M.D. Anderson experience. Radiother Oncol . 1999;52(2):173-178. doi:10.1016/s01678140(99)00070-5

5. Yahaya JJ, Odida M. Primary Osteosarcoma of the Breast with Extensive Chondroid Matrix in a Teenager Female Patient: The Paradoxical Diagnosis in Breast Mastopathy. Int Med Case Rep J . 2020;13:11-17. Published 2020 Jan 10. doi:10.2147/IMCRJ.S233674

6. Bahrami A, Resetkova E, Ro JY, Ibañez JD, Ayala AG. Primary osteosarcoma of the breast: report of 2 cases. Arch Pathol Lab Med . 2007;131(5):792-795. doi:10.1043/15432165(2007)131[792:POOTBR]2.0.CO;2

7. Milne DM, Sookar N, Umakanthan S, Rampersad F, Olivier L, Ali J. Primary Osteosarcoma of the Breast in a Patient Treated Previously for Invasive Ductal Carcinoma: An Unusual Presentation of a Very Rare Primary Breast Malignancy. Case Rep Surg . 2020;2020:1594127. Published 2020 Jan 16. doi:10.1155/2020/1594127

8. Yadav BS, Bansal A, Sharma SC, et al. A 62-year-old woman with osteogenic sarcoma in the contralateral breast 15 years after treatment for breast cancer. Semin Oncol . 2013;40(2):135-144. doi:10.1053/j.seminoncol.2013.01.010

9. Meunier B, Levêque J, Le Prisé E, Kerbrat P, Grall JY. Three cases of sarcoma occurring after radiation therapy of breast cancers. Eur J Obstet Gynecol Reprod Biol . 1994;57(1):33-36. doi:10.1016/00282243(94)90107-4

10. Alpert LI, Abaci IF, Werthamer S. Radiation-induced extraskeletal osteosarcoma. Cancer . 1973;31(6):1359-1363. doi:10.1002/1097-0142(197306)31:6<1359::aid-cncr2820310609>3.0.co;2-f

11. Remadi S, Doussis-Anagnostopoulu I, Mac Gee W. Primary osteosarcoma of the breast. Pathol Res Pract . 1995;191(5):471-477. doi:10.1016/S0344-0338(11)80737-5 
12. Jacob S, Japa D. Primary osteogenic sarcoma of the breast. Indian J Pathol Microbiol . 2010;53(4):785786. doi:10.4103/0377-4929.72090

13. Orta L, Suprun U, Goldfarb A, Bleiweiss I, Jaffer S. Radiation-associated extraskeletal osteosarcoma of the chest wall. Arch Pathol Lab Med . 2006;130(2):198-200. doi:10.1043/15432165(2006)130[198:REOOTC]2.0.CO;2

14. Vieira SC, Tavares MB, da Cruz Filho AJ, Sousa RB, Dos Santos LG. Metastatic osteosarcoma to the breast: a rare case. J Obstet Gynaecol Res . 2010;36(4):891-893. doi:10.1111/j.1447-0756.2010.01239.x

15. Momoi H, Wada Y, Sarumaru S, et al. Primary osteosarcoma of the breast. Breast Cancer . 2004;11(4):396-400. doi:10.1007/BF02968048

16. Hellmén E, Moller M, Blankenstein MA, Andersson L, Westermark B. Expression of different phenotypes in cell lines from canine mammary spindle-cell tumours and osteosarcomas indicating a pluripotent mammary stem cell origin. Breast Cancer Res Treat . 2000;61(3):197-210. doi:10.1023/a:1006410020384

17. Sarkar S, Kapur N, Mukri HM, Saurabh A, Kumar N. Chondroblastic osteosarcoma of breast in a case of phyllodes tumour with recurrence, a rare case report. Int J Surg Case Rep . 2016;27:189-191. doi:10.1016/j.ijscr.2016.08.035

18. Gafumbegete E, Fahl U, Weinhardt R, Respondek M, Elsharkawy AE. Primary osteosarcoma of the breast after complete resection of a metaplastic ossification: a case report. J Med Case Rep . 2016;10(1):231. Published 2016 Aug 17. doi:10.1186/s13256-016-1008-2

19. Guo W, Cao Y, Teng G, Liu J, Su J. Evolution and prognosis of breast osteosarcoma: A case report. Oncol Lett . 2016;11(1):789-791. doi:10.3892/ol.2015.3921

20. Yu SH, Noh WC, Bang HY, et al. Sarcoma and Sarcomatous Metaplastic Carcinoma of the Breast. Cancer Res Treat . 2001;33(6):463-468. doi:10.4143/crt.2001.33.6.463

21. Gonzalez-Licea A, Yardley JH, Hartmann WH. Malignant tumor of the breast with bone formation. Studies by light and electron microscopy. Cancer . 1967;20(8):1234-1247. doi:10.1002/10970142(196708)20:8<1234::aid-cncr2820200808>3.0.co;2-1

22. Alghofaily KA, Almushayqih MH, Alanazi MF, Salamah AAB, Benediktsson H. Primary Osteosarcoma of the Breast Arising in an Intraductal Papilloma. Case Rep Radiol . 2017;2017:5787829. doi:10.1155/2017/5787829

23. Crèvecoeur J, Jossa V, Gennigens C, Parmentier JC, Crèvecoeur A. Primary osteosarcoma of the breast: a case report. Clin Case Rep . 2015;4(1):62-66. Published 2015 Nov 23. doi:10.1002/ccr3.450

24. Tsubochi H, Sato N, Kaimori M, Imai T. Osteosarcomatous differentiation in lung metastases from a malignant phyllodes tumour of the breast. J Clin Pathol . 2004;57(4):432-434. doi:10.1136/jcp.2003.012336

25. Lin JJ, Huang CS, Yu J, et al. Malignant phyllodes tumors display mesenchymal stem cell features and aldehyde dehydrogenase/disialoganglioside identify their tumor stem cells. Breast Cancer Res . 2014;16(2):R29. Published 2014 Mar 26. doi:10.1186/bcr3631

26. Mačák J, Hurník P, Dvořáčková J, Mačáková J. An isolated metastasis to the heart from a malignant phyllodes tumor with osteosarcomatous differentiation. Cesk Patol . 2014;50(4):146-149.

27. Singhal V, Chintamani, Cosgrove JM. Osteogenic sarcoma of the breast arising in a cystosarcoma phyllodes: a case report and review of the literature. J Med Case Rep . 2011;5:293. doi:10.1186/17521947-5-293

28. Ribeiro-Silva A, Zambelli Ramalho LN, Zucoloto S. Phyllodes tumor with osteosarcomatous differentiation: a comparative immunohistochemical study between epithelial and mesenchymal cells. Tumori . 2006;92(4):340-346.

29. Graadt van Roggen JF, Zonderland HM, Welvaart K, Peterse JL, Hogendoorn PC. Local recurrence of a phyllodes tumour of the breast presenting with widespread differentiation to a telangiectatic osteosarcoma. J Clin Pathol . 1998;51(9):706-708. doi:10.1136/jcp.51.9.706

30. Silver SA, Tavassoli FA. Osteosarcomatous differentiation in phyllodes tumors. Am J Surg Pathol . 1999;23(7):815-821. doi:10.1097/00000478-199907000-00010

31. Bennett DL, Merenda G, Schnepp S, Lowdermilk MC. Primary breast osteosarcoma mimicking 
calcified fibroadenoma on screening digital breast tomosynthesis mammogram. Radiol Case Rep . 2017;12(4):648-652. Published 2017 Jul 29. doi:10.1016/j.radcr.2017.06.008

32. Bennett DL, Merenda G, Schnepp S, Lowdermilk MC. Primary breast osteosarcoma mimicking calcified fibroadenoma on screening digital breast tomosynthesis mammogram. Radiol Case Rep . 2017;12(4):648-652. Published 2017 Jul 29. doi:10.1016/j.radcr.2017.06.008

33. Krishnamurthy A. Primary breast osteosarcoma: A diagnostic challenge. Indian $J$ Nucl Med . 2015;30(1):39-41. doi:10.4103/0972-3919.147534

34. Ellmann A, Jawa ZM, Maharaj M. Primary osteogenic sarcoma of the breast detected on skeletal scintigraphy. Clin Nucl Med . 2006;31(8):474-475. doi:10.1097/01.rlu.0000227664.75779.38

35. Lumsden AB, Harrison D, Chetty U, Going JJ, Muir B. Osteogenic sarcoma-a rare primary tumour of the breast. Eur J Surg Oncol . 1985;11(2):183-186.

36. Ho JS, Rahmat K, Alli K, Wong KT, Yip CH. Primary breast osteosarcoma: dynamic contrastenhanced magnetic resonance imaging, proton spectroscopy and diffusion weighted imaging findings. Ann Acad Med Singap . 2012;41(10):473-475.

37. Zelek L, Llombart-Cussac A, Terrier P, et al. Prognostic factors in primary breast sarcomas: a series of patients with long-term follow-up. J Clin Oncol . 2003;21(13):2583-2588. doi:10.1200/JCO.2003.06.080

38. Kallianpur AA, Gupta R, Muduly DK, Kapali A, Subbarao KC. Osteosarcoma of breast: a rare case of extraskeletal osteosarcoma. J Cancer Res Ther . 2013;9(2):292-294. doi:10.4103/0973-1482.113392

39. Conde DM, Morais LC, Pacheco CF, et al. Primary osteosarcoma of the breast: pathological and imaging findings. Rev Assoc Med Bras (1992) . 2015;61(6):497-499. doi:10.1590/1806-9282.61.06.497

40. Szajewski M, Kruszewski WJ, Ciesielski M, Smiałek-Kusowska U, Czerepko M, Szefel J. Primary osteosarcoma of the breast: A case report. Oncol Lett . 2014;7(6):1962-1964. doi:10.3892/ol.2014.1981

41. Szajewski M, Kruszewski WJ, Ciesielski M, Smiałek-Kusowska U, Czerepko M, Szefel J. Primary osteosarcoma of the breast: A case report. Oncol Lett . 2014;7(6):1962-1964. doi:10.3892/ol.2014.1981

42. Khaldi L, Athanasiou ET, Hadjitheofilou CT. Primary mammary osteogenic sarcoma. Histol Histopathol . 2007;22(4):373-377. doi:10.14670/HH-22.373

43. Dragoumis D, Bimpa K, Assimaki A, Tsiftsoglou A. Primary osteogenic sarcoma of the breast. Singapore Med $J$. 2008;49(11):e315-e317.

44. Murakami S, Isozaki H, Shou T, et al. Primary osteosarcoma of the breast. Pathol Int . 2009;59(2):111115. doi:10.1111/j.1440-1827.2008.02338.x

45. Yoon CS, Kang SS. Primary osteosarcoma of the breast: a case report. Ann Surg Treat Res . 2017;93(1):57-60. doi:10.4174/astr.2017.93.1.57

46. Marinova L, Hadjieva T, Kanchev E, Vicheva S. Synchronous primary mammary osteosarcoma and invasive breast cancer. A case report - Pathohistological and immunohistochemical analysis. Rep Pract Oncol Radiother . 2014;20(1):72-76. Published 2014 May 1. doi:10.1016/j.rpor.2014.04.003

47. Vorobiof G, Hariparsad G, Freinkel W, Said H, Vorobiof DA. Primary osteosarcoma of the breast: a case report. Breast J . 2003;9(3):231-233. doi:10.1046/j.1524-4741.2003.09320.x

48. Zhao J, Zhang X, Liu J, Li J. Primary osteosarcoma of the breast with abundant chondroid matrix and fibroblasts has a good prognosis: A case report and review of the literature. Oncol Lett . 2013;6(3):745747. doi:10.3892/ol.2013.1446

49. Covello SP, Humphreys TR, Lee JB. A case of extraskeletal osteosarcoma with metastasis to the skin. $J$ Am Acad Dermatol . 2003;49(1):124-127. doi:10.1067/mjd.2003.297 